\title{
HER2 expression and relevant clinicopathological features in gastric and gastroesophageal junction adenocarcinoma in a Chinese population
}

Ling Shan, Jianming Ying ${ }^{*}$ and Ning Lu

\begin{abstract}
Background: With varied immunohistochemistry scoring criteria and patient cohorts, HER2-positivity rates in gastric cancer (GC) and gastroesophageal junction (GEJ) adenocarcinoma have been reported with a wide range. Recently standardized scoring criteria for GC and GEJ cancer has been established and recommended. In this study, the frequency of HER2 expression and the relationship between HER2 expression and clinicopathological features were examined in a large cohort of Chinese GC and GEJ cancer patients.

Methods: A total of 1463 patients, including 929 primary GCs and 534 primary GEJ adenocarcinomas, was retrospectively analyzed for HER2 overexpression by immunohistochemistry (IHC). Fluorescence in situ hybridization (FISH) analysis was used in 308 GCs and GEJ adenocarcinoma cases to assess HER2 gene amplification.

Results: HER2 overexpression (3+) was detected in 9.8\% of carcinomas and more frequently observed in GEJ cancer cases, in the intestinal type, and in the well or moderately differentiated type $(P=0.003,0.000$, and 0.000 , respectively). HER2 equivocal (2+) was detected in $14.4 \%$ of cases. As for the 308 cases analyzed by FISH, 39 (of 40, 97.5\%) IHC 3+ cases, 11 (of 38, 28.9\%) IHC 2+ cases, and 3 (of 230, 1.3\%) IHC 1+/0 cases showed HER2 gene amplification. A high concordance rate (98.5\%) between IHC and FISH was demonstrated.

Conclusions: Approximately 10\% of Chinese patients with primary GC and GEJ adenocarcinoma were HER2-positive on IHC. HER2 overexpression was associated with GEJ site, intestinal cancer subtype, and well or moderately differentiated carcinomas.

Virtual slides: The virtual slide(s) for this article can be found here: http://www.diagnosticpathology.diagnomx.eu/ vs/1935951199941072.
\end{abstract}

Keywords: HER2, Gastric cancer, Gastroesophageal junction adenocarcinoma, Chineses, Clinicopathological features

\section{Background}

Gastric cancer (GC) is the fourth most commonly diagnosed cancer and the second most common cause of cancer-related deaths worldwide [1]. It has been suggested that the incidence rate of gastroesophageal junction (GEJ) adenocarcinoma was increased in recent years $[2,3]$. Despite some advances in the prevention and treatment of gastric cancer, 5-year survival remains around $20 \%$ in most parts of the world. HER2 is now well recognized as a key factor in the development of certain

\footnotetext{
* Correspondence: jmying@hotmail.com

Department of Pathology, Cancer Hospital, Peking Union Medical College \& Chinese Academy of Medical Sciences, Beijing, China
}

solid human tumors, most notably in breast cancer. HER2 gene amplification and protein overexpression, which occur in $20 \%$ to $25 \%$ of breast cancer patients, have been recognized as prognostic and predictive markers for treatment [4]. Multiple detection methods have been established to examine HER2 gene status and protein expression [5-8]. Trastuzumab, a recombinant monoclonal antibody targeting HER2 protein, is now being applied not only in metastatic breast cancer cases but also to localized cases as adjuvant therapy $[9,10]$. A recent phase III randomized study (ToGA) revealed that combination treatment with trastuzumab and chemotherapy significantly improved survival in patients with advanced GC or GEJ cancer with HER2 overexpression [11]. Thus, trastuzumab 
was recently approved for the treatment of metastatic adenocarcinomas of the stomach and GEJ in many countries [12-17].

Although many studies have previously evaluated HER2 status in GC, the patient cohorts and scoring criteria have varied, resulting in discrepancies in HER2-positivity rates varying from about $4 \%$ to $53 \%$, with a median rate of $18 \%$ [18]. The ToGA study developed a new set of IHC scoring criteria based on the study by Hofmann et al. [19] and found HER2-positive (defined as IHC 3+ or IHC 2+/FISH+) tumors in $16 \%$ of metastatic GC cases. The efficacy of trastuzumab for treating metastatic GC with HER2 overexpression demonstrated in the ToGA study is also promising for resectable HER2-positive gastric cancer. However, few studies have been conducted to examine the frequency of HER2-positive tumors determined by the new criteria in resectable gastric cancer [20,21], especially in a large Chinese cohort. In this study, IHC analysis according to standardized scoring criteria was used to assess the incidence of HER2-positivity in primary resected GC and GEJ cancer samples in a C9pt?>The relationship between HER2 overexpression and gene amplification was also examined in GC and GEJ adenocarcinoma.

\section{Methods}

\section{Samples}

A total of 1,463 patients with primary GC or GEJ adenocarcinoma, who received curative surgery (no history of neoadjuvant chemotherapy) in the Cancer Institute \& Hospital, Chinese Academy of Medical Sciences (CICAMS), Beijing, China, between August 2009 and February 2012, was included in this retrospective study. All tumor samples were fixed in $10 \%$ neutral buffered formalin for 24-48 h and embedded in paraffin, and routinely diagnosed in the Department of Pathology, CICAMS, Beijing. The study protocol was approved by the Institutional Review Board (IRB). The patients' medical records were reviewed to obtain patients' clinicopathological parameters, including age at diagnosis, sex, tumor location, histological classification, and pathological TNM stage. Histological classification was determined according to the World Health Organization (WHO) classification and Lauren's classification.

\section{Immunohistochemistry}

Automated IHC was performed on 4- $\mu$ m-thick sections using an automated slide stainer, the Ventana Benchmark XT (Ventana Medical Systems, Tucson, AZ, USA), according to the manufacturer's instructions, for the Ventana CONFIRM ${ }^{\mathrm{m}}$ HER2/neu (4B5) Rabbit Monoclonal Primary Antibody. HER2 IHC was scored using the scoring scheme proposed by Hofmann et al. [19] in the ToGA cohort of gastric cancer (ToGA score) and Ruschoff et al. [22] as follows: 0, no staining or membranous reactivity in $<10 \%$ of tumor cells; $1+$, faint/ barely perceptible membranous reactivity in $\geq 10 \%$ of tumor cells (cells are reactive only in part of their membrane); $2+$, weak to moderate complete, basolateral, or lateral membranous reactivity in $\geq 10 \%$ of tumor cells; and $3+$, complete, basolateral, or lateral membranous reactivity of strong intensity in $\geq 10 \%$ of tumor cells.

Samples scoring IHC 0 or IHC 1+ were considered negative for HER2 overexpression, whereas samples scoring IHC 3+ were considered positive for HER2 overexpression. Samples scoring IHC 2+ were considered equivocal for HER2 overexpression.

\section{Fluorescence in situ hybridization}

Fluorescence in situ hybridization (FISH) analysis was carried out with the PathVysion HER2 DNA probe kit and procedures (Vysis/Abbott, Abbott Park, IL, USA). The kit contains two fluorochrome-labeled DNA probes, LSI HER2 (labeled with SpectrumOrange) and CEP17 (chromosome 17 enumeration probe, labeled with SpectrumGreen). Pretreatment was carried out with the Paraffin Pretreatment Kit (VysisAbbott). The HER2 signals and CEP17 signals of 20 nuclei of invasive tumor cells in two different areas were counted using a Zeiss AxioImager M2 epifluorescence microscope (Carl Zeiss, Oberkochen, Germany) equipped with an $\times 100$ oil immersion objective and 4,6'-diamidino-2phenylindole (DAPI)/Spectrum Green/Orange single and triple bandpass filters. The HER2/chromosome 17 ratios were interpreted as follows: a HER2/CEP17 ratio higher than 2.2 was defined as amplification of the HER2 gene, while a ratio $<1.8$ was defined as no amplification of the HER2 gene. When the ratio was between 1.8 and 2.2, signals in another 20 nuclei were counted, and the HER2/ CEP17 ratio in a total of 40 nuclei was determined. When the ratio was $\geq 2.0$, it was defined as amplification of the HER2 gene; otherwise it was defined as no amplification of the HER2 gene.

\section{Statistical analysis}

Statistical analysis was performed using the chi-square test to analyze associations between HER2 status and clinicopathological parameters. A $P$ value less than 0.05 was considered significant. Data were analyzed using the SPSS statistical software program for Microsoft Windows (SPSS Inc., Chicago, IL, USA).

\section{Results}

\section{HER2 overexpression and clinicopathological} characteristics

A total of 1,463 patients was evaluated by IHC analysis. The patients' clinicopathological characteristics are listed in Table 1. Their median age was 58 years (range, 20 to 82 years), with a male predominance $(75.5 \%)$. Of all patients, there were 650 cases $(44.4 \%)$ of intestinal type by Lauren's classification, 564 cases (38.6\%) of diffused type, 
Table 1 The correlation between HER2 expression status and clinicopathological parameters

\begin{tabular}{|c|c|c|c|c|c|}
\hline & Overall & $\begin{array}{l}\text { HER2 } \\
\text { Overexpression }\end{array}$ & $\begin{array}{l}\text { HER2- } \\
\text { Equivocal }\end{array}$ & $\begin{array}{l}\text { HER2- } \\
\text { Negative }\end{array}$ & $P$ value \\
\hline Pathologic feature & $n=1463$ & $n=143(\%)$ & $\mathrm{n}=211(\%)$ & $n=1109(\%)$ & \\
\hline Age at diagnosis & & & & & 0.102 \\
\hline$\geq 60$ years & 683 & $80(11.7)$ & $108(15.8)$ & $495(72.5)$ & \\
\hline$<60$ years & 780 & $63(8.1)$ & $103(13.2)$ & $614(78.7)$ & \\
\hline Sex & & & & & 0.344 \\
\hline Male & 1104 & $109(9.9)$ & $171(15.5)$ & $824(74.6)$ & \\
\hline Female & 359 & $34(9.5)$ & $40(11.1)$ & $285(79.4)$ & \\
\hline Tumor location & & & & & 0.003 \\
\hline GC & 929 & $65(7.0)$ & $121(13.0)$ & $743(80.0)$ & \\
\hline GEJ & 534 & $78(14.6)$ & $90(16.9)$ & $366(68.5)$ & \\
\hline \multicolumn{6}{|l|}{ Tumor subtype } \\
\hline (Lauren classification) & & & & & 0.000 \\
\hline Intestinal & 650 & $109(16.8)$ & $111(17.1)$ & $430(66.2)$ & \\
\hline Diffuse & 564 & $13(2.3)$ & $63(11.2)$ & $488(86.5)$ & \\
\hline Mixed & 249 & $21(8.4)$ & $37(14.9)$ & $191(76.7)$ & \\
\hline Tumor differentiation & & & & & 0.000 \\
\hline Well & 25 & $4(16)$ & $9(36)$ & $12(48)$ & \\
\hline Moderately & 369 & $74(20.1)$ & $70(19.0)$ & $225(61.0)$ & \\
\hline Poorly & 1069 & $65(6.1)$ & $132(12.3)$ & $872(81.6)$ & \\
\hline pT status & & & & & 0.053 \\
\hline pT1 & 206 & $16(7.8)$ & $33(16.0)$ & $157(76.2)$ & \\
\hline pT2 & 147 & $23(15.6)$ & $28(19.0)$ & $96(65.3)$ & \\
\hline pT3 & 1074 & $102(9.5)$ & $145(13.5)$ & $827(77.0)$ & \\
\hline pT4 & 36 & $2(5.6)$ & $5(13.9)$ & 29 (80.6) & \\
\hline pN status & & & & & 0.074 \\
\hline pNO & 411 & $39(9.5)$ & $66(16.1)$ & $306(74.5)$ & \\
\hline pN1 & 235 & 27 (11.5) & $42(17.9)$ & $166(70.6)$ & \\
\hline pN2 & 306 & $20(6.5)$ & $42(13.7)$ & $244(79.7)$ & \\
\hline pN3 & 511 & $57(11.2)$ & $61(11.9)$ & $393(76.9)$ & \\
\hline pM status & & & & & 0.571 \\
\hline pMO & 1429 & $140(9.8)$ & $119(8.3)$ & $1080(75.6)$ & \\
\hline pM1 & 34 & $3(8.8)$ & $2(5.9)$ & 29 (85.3) & \\
\hline pTNM stages & & & & & 0.327 \\
\hline IA & 162 & $14(8.6)$ & 29 (17.9) & $119(73.5)$ & \\
\hline $\mathrm{IB}$ & 93 & $8(8.6)$ & $17(18.3)$ & $68(73.1)$ & \\
\hline$\| \mathrm{A}$ & 201 & $25(12.4)$ & $33(16.4)$ & $143(71.1)$ & \\
\hline$\| B$ & 230 & 25 (10.9) & $34(14.8)$ & $171(74.3)$ & \\
\hline$\| I A$ & 264 & $15(5.7)$ & $37(14.8)$ & $212(80.3)$ & \\
\hline$\| \mathrm{IIB}$ & 455 & $51(11.2)$ & $56(12.3)$ & $348(76.5)$ & \\
\hline IIIC & 24 & $2(8.3)$ & $3(12.5)$ & 19 (79.2) & \\
\hline IV & 34 & $3(8.8)$ & $2(5.9)$ & $29(85.3)$ & \\
\hline
\end{tabular}


and 249 cases $(17 \%)$ of mixed type. In terms of tumor location, approximately two-thirds of tumors were primary gastric cancers (63.5\%), and one-third was primary GEJ adenocarcinomas (36.5\%). The tumors were poorly differentiated in $73.1 \%$, moderately differentiated in $25.2 \%$, and well-differentiated in $1.7 \%$. Overall, most patients were at pathologic stage T3 (73.4\%), with $14.1 \%$, $10 \%$, and $2.5 \%$ at stage $\mathrm{T} 1, \mathrm{~T} 2$, and $\mathrm{T} 4$, respectively. Regarding the pathologic $\mathrm{N}$ stage, there was an approximately equal distribution of patients at N0, N1, N2, or N3 stages $(28.1 \%, 16.1 \%, 20.9 \%, 34.9 \%$, respectively). The majority of patients $(97.7 \%)$ had no distant metastases.

Of the 1463 patients, $143(9.8 \%)$ patients were scored as HER2 overexpression (3+), while 211 (14.4\%) and 1109 (1463, 75.8\%) were equivocal and negative, respectively. Table 1 summarizes HER2 expression status by subgroup. HER2 was overexpressed (score 3+) in 7.0\% (65/929) of GC cases and $14.6 \%(78 / 534)$ of GEJ adenocarcinoma cases (Figure 1A). Equivocal expression (2+) of HER2 protein was observed in 13.0\% (121/929) of GC cases and $16.9 \%(90 / 534)$ of GEJ adenocarcinoma cases (Figure 1B). HER2 was negative $(1+/ 0)$ in $80.0 \%(743 / 929)$ of GC cases and $68.5 \%(366 / 534)$ of GEJ adenocarcinoma samples (Figure $1 \mathrm{C}$ and D). A significant difference in HER2 overexpression was found between GCs and GEJ adenocarcinomas $(7.0 \%$ vs. $14.6 \%, P<0.05)$. HER2 overexpression was associated with the intestinal-type carcinomas by the Lauren classification $(P<0.001): 16.8 \%$ in intestinal vs. $2.3 /$ $8.4 \%$ in diffuse-/mixed-type cancers. HER2 overexpression was also correlated with well or moderately differentiated carcinomas according to the WHO classification. No correlation was observed between HER2 overexpressed and not overexpressed cases in terms of age, sex, pT, N, M factors, or pathologic TNM stage.

\section{Concordance of HER2 IHC and FISH}

FISH analysis was performed in 308 randomly selected GC and GEJ adenocarcinoma cases, including 40 cases with
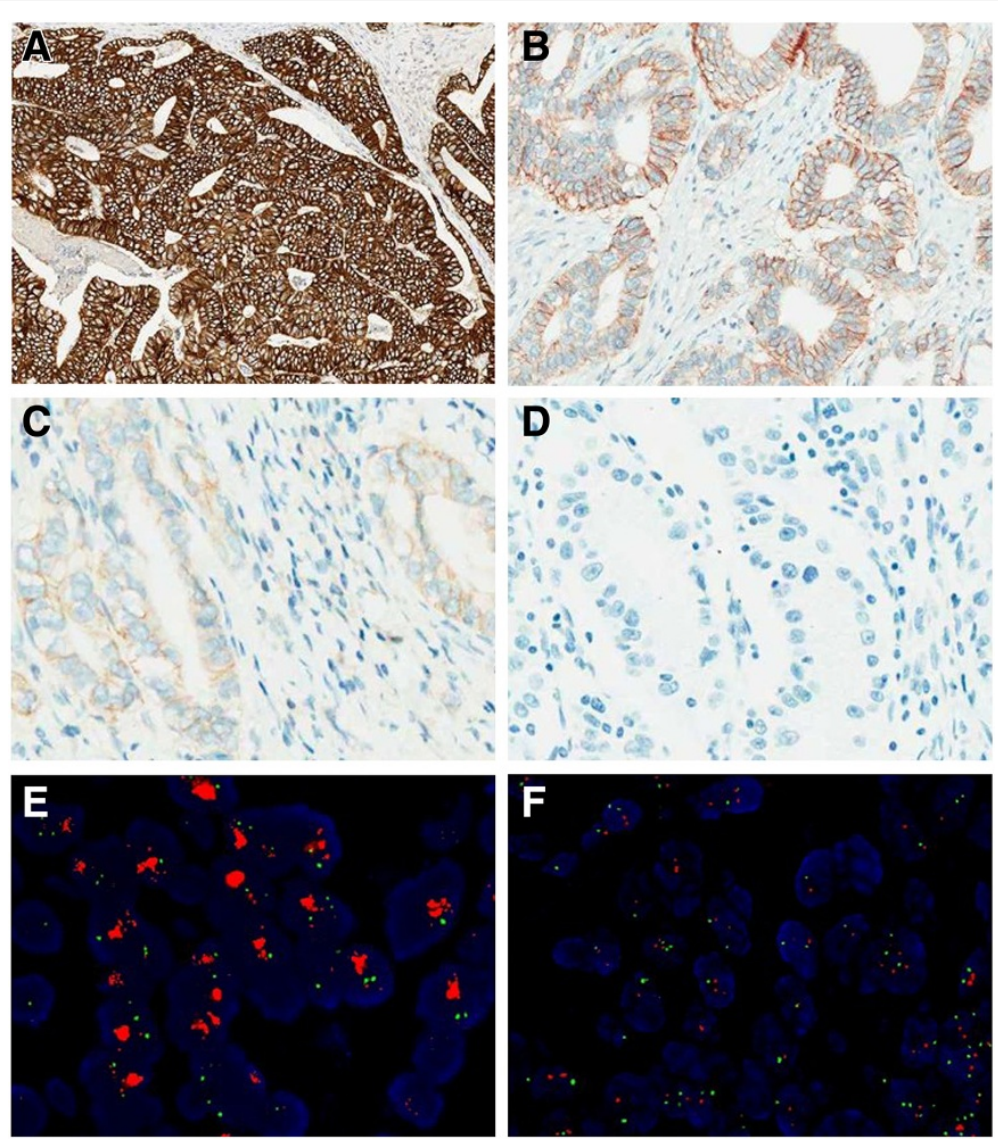

Figure 1 Representative cases of IHC staining and FISH analysis in GC and GEJ adenocarcinoma. A. HER2 IHC staining shows complete, basolateral, or lateral membranous reactivity of strong intensity (3+) in tumor cells (original magnification, $\times 40$ ). B. HER2 IHC staining shows weak to moderate complete, basolateral, or lateral membranous reactivity (2+) in tumor cells (original magnification, $\times 100$ ). C. faint/barely perceptible membranous reactivity $(1+)$ in tumor cells (original magnification, $\times 200$ ). D. no staining (0) in tumor cells (original magnification, $\times 200$ ). E. a GC case with HER2 amplification using FISH analysis. Green signals refer to the reference probe of Chr. 17 centromere, while red signals are the target probe for HER2. F. A GC case without HER2 amplification using FISH analysis. 
HER2 overexpression (IHC $3+$ ), 38 cases with equivocal (IHC 2+), and 230 cases with negative (IHC 1+/0) HER2 expression. HER2 amplification was observed in $97.5 \%$ (39/40) of HER2 IHC $3+$ cases, $28.9 \%(11 / 38)$ of HER2 IHC $2+$ cases, and 1.3\% (3/230) of HER2-negative $(1+/ 0)$ cases, showing a concordance rate of $98.5 \%$ between IHC and FISH (Table 2) (Figure 1E and F).

\section{Discussion}

In this study, 143 of 1463 (9.8\%) of GC and GEJ adenocarcinoma cases were HER2-positive $(3+)$ by IHC in one of the largest Chinese studies to date. In the ToGA trial, the percentage of HER2-positive (IHC $3+$ or IHC $2+/ F I S H$ positive) GC or GEJ cancer patients was $22.1 \%$ overall and around $10.4 \%$ of IHC $3+$ in resected samples [23], similar to the present result. Recent studies also reported a range of $8.5 \%$ to $10.3 \%$ for HER2 overexpression in GC $[20,21,24]$. Recently, three studies in Chinese GC cases applied the same FDA-approved reagents and scoring criteria and reported HER2 IHC 3+ rates of 9.0\% (77/860) [25], 6.9\% (10/145) [26], and 5.8\% (4/69) [27], respectively, all slightly lower than the present result. This variation may be partly explained by different sample sets. In addition, HER2-positivity varied by tumor site, with higher rates of HER2-positivity in GEJ adenocarcinoma than in stomach cancer in this study $(14.6 \%$ vs. $7.0 \%$ respectively; $P<0.01$ ), which is consistent with the results of other studies $[11,20]$. In the ToGA trial, the countries with the highest ratio of GEJ:stomach cancer were found to have aboveaverage HER2-positivity rates, regardless of sample size [23]. Another explanation is the different ratio of intestinal:diffuse/ mixed cancer among the studies. A positive association between HER2-positivity and intestinal-type cancers was identified in this and other studies [28-33]. In the ToGA trial, for example, countries with higher ratios of intestinal:diffuse/ mixed cancer had increased HER2-positivity rates [23].

No correlation was found between HER2 overexpression and $\mathrm{pT}, \mathrm{N}$, or $\mathrm{M}$ factors, or TNM stages, in the present study. Previous studies including all pathological stages have also reported no correlation between pathological stage and overexpression [20,21,24]. A correlation of HER2-positivity with well or moderately differentiated carcinomas was found in the present study $(P<0.01)$. However, other studies demonstrated both an association and

Table 2 Correlation between HER2 overexpression and gene amplification in GC and GEJ adenocarcinoma

\begin{tabular}{|c|c|c|c|c|}
\hline \multirow[b]{2}{*}{ FISH } & \multicolumn{2}{|r|}{ IHC } & \multirow[b]{2}{*}{$1+/ 0$} & \multirow[b]{2}{*}{ Tota } \\
\hline & $3+$ & $2+$ & & \\
\hline Amplification & $39(97.5 \%)$ & $11(28.9 \%)$ & $3(1.3 \%)$ & 53 \\
\hline No amplification & $1(2.5 \%)$ & 27 (71.1\%) & 227 (98.7\%) & 255 \\
\hline Total & 40 & 38 & 230 & 308 \\
\hline
\end{tabular}

no association between HER2 overexpression and tumor differentiation $[25,32,34,35]$. These conflicting data may be due to different sample sizes and the low prevalence of HER2 in GC and GEJ adenocarcinoma. In addition, varying methods of evaluation and scoring schemes with different cut-points were used before the establishment of a standard guideline for assessing HER2 expression. Perhaps with the current consistent guidelines for HER2 assessment in this disease, future studies will provide more clarity regarding this issue.

The present study revealed a high concordance rate, 98.5\%, between IHC and FISH, which is similar to another study of surgical resections from 145 Chinese GC cases, with a concordance rate of $94.5 \%$ [26]. In contrast, in the ToGA study, the concordance rate of IHC and FISH was $87.2 \%$, as $7.5 \%$ of IHC $1+/ 0$ samples and $54.6 \%$ of IHC 2+ cases were found to be FISH-positive [23]. One of the possible explanations for this discrepancy might be different specimen types and reagents. In the ToGA study, both biopsies and surgically-resected specimens were included; the HER2-positivity rate was higher in biopsies than in surgically-resected specimens $(23.1 \%$ vs. $19.9 \% ; \mathrm{P}=0.03)$, and biopsy samples were also more likely to be HER2-amplified than surgical samples when analyzed by FISH $(\mathrm{P}=0.01)$ than by IHC [23]. The majority of current studies using a standard guideline for HER2 expression assessment used biopsies and tissue microarrays (TMAs), which were tested analogous to biopsies, to interrogate HER status in GC. The concordance rate of IHC and FISH in these studies varied from $88.6 \%$ to $97.7 \%$ [20,32,36]. A specific study evaluating HER2 protein expression on whole-tissue sections and TMAs was conducted, indicating a discrepancy in HER2 expression on whole-tissue sections from TMAs. For instance, the proportion of IHC $2+$ was $4.7 \%$ in wholetissue sections, whereas it was $8.6 \%$ in TMAs, which might be partially explained by heterogeneous HER2 expression [33]. Another possibility, which was revealed by a study aiming to validate the guideline for HER2expression assessment in GC, was different interpretations of staining of TMAs among observers, although a uniform assessment guideline was followed [22]. Therefore, reliable separation of IHC $1+/ 0$ and IHC $2+$ may be difficult in biopsy samples, and FISH analysis should be used for definitive classification.

In conclusion, a thorough analysis of 1463 Chinese GC/GEJ cancer cases using FDA-approved reagents showed HER2 overexpression in $9.8 \%$ of carcinomas, with a strong preference for GEJ location, intestinal cancer subtype, and well or moderately differentiated carcinomas. Approximately $29 \%$ of IHC $2+$ cases showed HER2 gene amplification, and there was a high concordance rate $(98.5 \%)$ between $\mathrm{IHC}$ and FISH in GC and GEJ adenocarcinoma. 


\section{Competing interests}

None of the authors has an affiliation or conflict of interest.

\section{Authors' contributions}

Study concept and design: JY, NL. Analysis and interpretation of data: LS, JY Drafting of the manuscript: LS, JY. All authors read and approved the final manuscript.

\section{Acknowledgements}

This study was supported by grants from the Beijing Hope Run Special Fund (LC2012A08) and the National Natural Science Foundation of China (30801344).

Received: 11 March 2013 Accepted: 22 April 2013

Published: 9 May 2013

\section{References}

1. Kamangar F, Dores GM, Anderson WF: Patterns of cancer incidence, mortality, and prevalence across five continents: defining priorities to reduce cancer disparities in different geographic regions of the world. J Clin Oncol 2006, 24(14):2137-2150.

2. Abdi-Rad A, Ghaderi-sohi S, Nadimi-Barfroosh H, Emami S: Trend in incidence of gastric adenocarcinoma by tumor location from 1969-2004: a study in one referral center in Iran. Diagn Pathol 2006, 1:5.

3. Botterweck AA, Schouten LJ, Volovics A, Dorant E, van Den Brandt PA: Trends in incidence of adenocarcinoma of the oesophagus and gastric cardia in ten European countries. Int J Epidemiol 2000, 29(4):645-654.

4. Slamon DJ, Clark GM, Wong SG, Levin WJ, Ullrich A, McGuire WL: Human breast cancer: correlation of relapse and survival with amplification of the HER-2/neu oncogene. Science 1987, 235(4785):177-182.

5. Perez EA, Press MF, Dueck AC, Jenkins RB, Kim C, Chen B, Villalobos I, Paik S, Buyse $M$, Wiktor $A E$, et al: Immunohistochemistry and fluorescence in situ hybridization assessment of HER2 in clinical trials of adjuvant therapy for breast cancer (NCCTG N9831, BCIRG 006, and BCIRG 005). Breast Canc Res Treat 2013, 138(1):99-108.

6. Kiyose S, Igarashi H, Nagura K, Kamo T, Kawane K, Mori H, Ozawa T, Maeda $\mathrm{M}$, Konno K, Hoshino $\mathrm{H}$, et al: Chromogenic in situ hybridization (CISH) to detect HER2 gene amplification in breast and gastric cancer: comparison with immunohistochemistry (IHC) and fluorescence in situ hybridization (FISH). Pathol Int 2012, 62(11):728-734.

7. Nitta H, Kelly BD, Padilla M, Wick N, Brunhoeber P, Bai I, Singh S, Ranger-Moore J, Bieniarz C, Tsuda H, Grogan TM: A gene-protein assay for human epidermal growth factor receptor 2 (HER2): brightfield tricolor visualization of HER2 protein, the HER2 gene, and chromosome 17 centromere (CEN17) in formalin-fixed, paraffin-embedded breast cancer tissue sections. Diagn Pathol 2012, 7:60.

8. Nitta H, Hauss-Wegrzyniak B, Lehrkamp M, Murillo AE, Gaire F, Farrell M, Walk E, Penault-Llorca F, Kurosumi M, Dietel M, et al: Development of automated brightfield double in situ hybridization (BDISH) application for HER2 gene and chromosome 17 centromere (CEN 17) for breast carcinomas and an assay performance comparison to manual dual color HER2 fluorescence in situ hybridization (FISH). Diagn Pathol 2008, 3:41.

9. Slamon DJ, Leyland-Jones B, Shak S, Fuchs H, Paton V, Bajamonde A Fleming T, Eiermann W, Wolter J, Pegram M, et al: Use of chemotherapy plus a monoclonal antibody against HER2 for metastatic breast cancer that overexpresses HER2. N Engl J Med 2001, 344(11):783-792.

10. Smith I, Procter M, Gelber RD, Guillaume S, Feyereislova A, Dowsett M, Goldhirsch A, Untch M, Mariani G, Baselga J, et al: 2-year follow-up of trastuzumab after adjuvant chemotherapy in HER2-positive breast cancer: a randomised controlled trial. Lancet 2007, 369(9555):29-36.

11. Bang YJ, Van Cutsem E, Feyereislova A, Chung HC, Shen L, Sawaki A, Lordick F, Ohtsu A, Omuro Y, Satoh T, et al: Trastuzumab in combination with chemotherapy versus chemotherapy alone for treatment of HER2-positive advanced gastric or gastro-oesophageal junction cancer (ToGA): a phase 3, open-label, randomised controlled trial. Lancet 2010, 376(9742):687-697.

12. EMEA and European Medicines Agency O; 2009. www.emea.europa.eu/ docs/en_GB/document_library/Summary_of_opinion/human/000278/ WC500059913.pdf.

13. Phillips BE, Tubbs RR, Rice TW, Rybicki LA, Plesec T, Rodriguez CP, Videtic GM, Saxton JP, Ives DI, Adelstein DJ: Clinicopathologic features and treatment outcomes of patients with human epidermal growth factor receptor 2-positive adenocarcinoma of the esophagus and gastroesophageal junction. Dis Esophagus 2013, 26(3):299-304.

14. Choi JH, Han HS, Lee HC, Lee OJ, Kim JT, Lim SN, Lee KH, Kim ST: Positive response to trastuzumab in a case of HER2-overexpressing metastatic gastric cancer that presented as severe thrombocytopenia. Onkologie 2011, 34(11):621-624.

15. Weissinger F, Reymond M, Dumke K, Kruger M: Successful treatment of a patient with HER2-positive metastatic gastric cancer with third-line combination therapy with irinotecan, 5-fluorouracil, leucovorin and trastuzumab (FOLFIRI-T). Onkologie 2011, 34(10):548-551.

16. Gravalos C, Gomez-Martin C, Rivera F, Ales I, Queralt B, Marquez A, Jimenez U, Alonso V, Garcia-Carbonero R, Sastre J, et al: Phase II study of trastuzumab and cisplatin as first-line therapy in patients with HER2-positive advanced gastric or gastroesophageal junction cancer. Clin Transl Oncol 2011, 13(3):179-184.

17. Shitara K, Mizota A, Yatabe Y, Kondo C, Nomura M, Yokota T, Takahari D, Ura T, Muro K: Lapatinib plus trastuzumab for a patient with heavily pretreated gastric cancer that progressed after trastuzumab. Jpn J Clin Oncol 2011, 41(5):663-665.

18. Chua TC, Merrett ND: Clinicopathologic factors associated with HER2-positive gastric cancer and its impact on survival outcomes-A systematic review. Int J Canc 2012, 130(12):2845-2856.

19. Hofmann M, Stoss O, Shi D, Buttner R, van de Vijver M, Kim W, Ochiai A, Ruschoff J, Henkel T: Assessment of a HER2 scoring system for gastric cancer: results from a validation study. Histopathology 2008, 52(7):797-805.

20. Kunz PL, Mojtahed A, Fisher GA, Ford JM, Chang DT, Balise RR, Bangs CD Cherry AM, Pai RK: HER2 expression in gastric and gastroesophageal junction adenocarcinoma in a US population: clinicopathologic analysis with proposed approach to HER2 assessment. Appl Immunohistochem Mol Morphol 2012, 20(1):13-24.

21. Kataoka Y, Okabe H, Yoshizawa A, Minamiguchi S, Yoshimura K, Haga H, Sakai Y: HER2 expression and its clinicopathological features in resectable gastric cancer. Gastric Canc 2012, 16(1):84-93.

22. Ruschoff J, Dietel M, Baretton G, Arbogast S, Walch A, Monges G, Chenard MP, Penault-Llorca F, Nagelmeier I, Schlake W, et al: HER2 diagnostics in gastric cancer-guideline validation and development of standardized immunohistochemical testing. Virchows Arch 2010, 457(3):299-307.

23. Bang Y, Chung H, Xu J, Lordick F, Sawaki A, Al-Sakaff N, Lipatov O, See C, Rueschoff J, Van Cutsem E: Pathological features of advanced gastric cancer (GC): Relationship to human epidermal growth factor receptor 2 (HER2) positivity in the global screening programme of the ToGA trial. J Clin Oncol 2009, 27(15S):4556.

24. Bozzetti C, Negri FV, Lagrasta CA, Crafa P, Bassano C, Tamagnini I, Gardini G, Nizzoli R, Leonardi F, Gasparro D, et al: Comparison of HER2 status in primary and paired metastatic sites of gastric carcinoma. Br J Canc 2011, 104(9):1372-1376.

25. Wu HM, Liu YH, Lin F, Xu FP, Luo DL, Zhang F, Zhuang HG, Luo XL, Wu WL, Lin XT: Lin XT: [Association of HER2 protein expression with clinicopathologic features and prognosis in Chinese patients with gastric carcinoma]. Zhonghua Bing Li Xue Za Zhi 2011, 40(5):296-299.

26. Yan SY, Hu Y, Fan JG, Tao GQ, Lu YM, Cai X, Yu BH, Du YQ: Clinicopathologic significance of HER-2/neu protein expression and gene amplification in gastric carcinoma. World J Gastroenterol 2011, 17(11):1501-1506.

27. Wang YK, Gao CF, Yun T, Chen Z, Zhang XW, Lv XX, Meng NL, Zhao WZ: Assessment of ERBB2 and EGFR gene amplification and protein expression in gastric carcinoma by immunohistochemistry and fluorescence in situ hybridization. Mol Cytogenet 2011, 4(1):14.

28. Grabsch H, Sivakumar S, Gray S, Gabbert HE, Muller W: HER2 expression in gastric cancer: Rare, heterogeneous and of no prognostic value - conclusions from 924 cases of two independent series. Cell Oncol 2010, 32(1-2):57-65.

29. Marx AH, Tharun L, Muth J, Dancau AM, Simon R, Yekebas E, Kaifi JT, Mirlacher M, Brummendorf TH, Bokemeyer C, et al: HER-2 amplification is highly homogenous in gastric cancer. Hum Pathol 2009, 40(6):769-777.

30. Barros-Silva JD, Leitao D, Afonso L, Vieira J, Dinis-Ribeiro M, Fragoso M, Bento MJ, Santos L, Ferreira P, Rego S, et al: Association of ERBB2 gene status with histopathological parameters and disease-specific survival in gastric carcinoma patients. Br J Canc 2009, 100(3):487-493. 
31. Zhang XL, Yang YS, Xu DP, Qu JH, Guo MZ, Gong Y, Huang J: Comparative study on overexpression of HER2/neu and HER3 in gastric cancer. World J Surg 2009, 33(10):2112-2118.

32. Tafe $L$, Janjigian YY, Zaidinski M, Hedvat CV, Hameed MR, Tang LH, Hicks JB, Shah MA, Barbashina V: Human epidermal growth factor receptor 2 testing in gastroesophageal cancer: correlation between immunohistochemistry and fluorescence in situ hybridization. Arch Pathol Lab Med 2011, 135(11):1460-1465.

33. Kim KC, Koh YW, Chang HM, Kim TH, Yook JH, Kim BS, Jang SJ, Park YS: Evaluation of HER2 protein expression in gastric carcinomas: comparative analysis of 1,414 cases of whole-tissue sections and 595 cases of tissue microarrays. Ann Surg Oncol 2011, 18(10):2833-2840

34. Yu GZ, Chen Y, Wang JJ: Overexpression of Grb2/HER2 signaling in Chinese gastric cancer: their relationship with clinicopathological parameters and prognostic significance. J Cancer Res Clin Oncol 2009, 135(10):1331-1339.

35. Zhan GH: Multicenter study on HER-2/neu gene amplification and protein expression in patients with gastric cancer. Chin J Dig 2006 26(10):657-660.

36. Yan B, Yau EX, Bte Omar SS, Ong CW, Pang B, Yeoh KG, Salto-Tellez M: A study of HER2 gene amplification and protein expression in gastric cancer. J Clin Pathol 2010, 63(9):839-842.

\section{Submit your next manuscript to BioMed Central and take full advantage of:}

- Convenient online submission

- Thorough peer review

- No space constraints or color figure charges

- Immediate publication on acceptance

- Inclusion in PubMed, CAS, Scopus and Google Scholar

- Research which is freely available for redistribution 